\title{
Sputtering of Mo and Ag with Xenon lons from a Radio-Frequency Ion Thruster
}

\author{
L. J. Buntrock, ${ }^{1, \text { a) }}$ C. Volkmar, ${ }^{2, \text { b) }}$ and K. Hannemann ${ }^{1, c)}$ \\ ${ }^{1)}$ Spacecraft Department, German Aerospace Center (DLR), Bunsenstraße 10, 37073 Göttingen, \\ Germany \\ ${ }^{2)}$ Technische Hochschule Mittelhessen - University of Applied Sciences, Competence Center for \\ Nanotechnology and Photonics (NanoP) - Space Electronics Workgroup, Wiesenstrasse 14, 35390 Giessen, \\ Germany
}

(Dated: 30 March 2021)

The goal of this work was the setup of an electric propulsion (EP) sputtering test section as a feasibility study for ground-based sputter testing of spacecraft materials with a radio-frequency ion thruster (RIT). Such experiments deliver valuable data which are scarce but highly desired to model EP-based space missions, for example with SPIS (Spacecraft Plasma Interaction System), in order to predict the performance and lifetime of spacecraft components. This study assessed if sufficient testing conditions can be met to produce reliable experimental material data in the future. Therefore, the thruster was operated at ion energies of $1.5 \mathrm{keV}$ and $1.8 \mathrm{keV}$, and a quartz crystal microbalance (QCM) was installed to detect sputter deposition rates. Molybdenum (Mo) and silver (Ag) were chosen as sputter targets. Wafer substrates served as a passive sampling method to characterize the composition of sputtered material by Rutherford Backscattering Spectrometry (RBS). Additionally, sputtering simulations matching the experimental conditions were conducted with the software SDTrimSP. We obtained comparable experimental and computational data, as measured sputter deposition rates lie within the simulated order of magnitude and to some extent show the predicted angular dependence. Analysis of deposited sputter material revealed the formation of metal oxides which requires a future adaption of the material specific QCM settings. Furthermore, the cooling system of the QCM sensor head was not sufficient, limiting the comparability of results.

\section{INTRODUCTION}

Today, electric propulsion (EP) is part of nearly every space mission. Especially if long mission duration and comparably low propellant consumption go hand in hand which is primarily the case for telecommunication satellites electric thrusters offer inherent advantages over their chemical pendants. Those advantages are based on a comparably very high specific impulse $I_{\text {sp }}$. Additionally, EP offers high scalability of thrust levels, enabling multiple maneuvers with only one type of thruster (e.g. electric orbit raising (EOR) followed by station-keeping $(\mathrm{SK})$ ). Detailed information on EP (especially on the two major concepts Hall and ion thrusters) can be found in ${ }^{1}$ and $^{2}$, respectively.

To ensure long mission duration, not only propellant consumption plays a role. Erosion and degradation of thruster components often define the lifetime of a thruster. Especially gridded ion thrusters such as radio-frequency (RF) ion thrusters (RIT) suffer from severe degradation of the extraction grid system ${ }^{3,4}$. In the context of EP, nearly every degrading effect is caused by material sputtering due to high-energetic ion bombardment. On a larger scale, those sputtering effects also are present due to ion beam (plume) divergence. Most prominently, EP may damage solar panels or other satellite components in the vicinity of the thrusters. To put it crudely, the effects of contamination in the context

\footnotetext{
a) Author to whom correspondence should be addressed: leonie.buntrock@dlr.de

b) Electronic mail: chris.volkmar@ei.thm.de

c)Electronic mail: klaus.hannemann@dlr.de
}

of chemical propulsion ${ }^{5}$ are similar to those of sputtering in the context of electric propulsion.

During EP mission design, those sputtering effects are taken into account using special frameworks such as ESA's SPIS (Spacecraft Plasma Interaction System). In theory, the upper mentioned contamination effects can be studied as functions of the points of thruster operation, thrust vector, geometry of thruster, satellite and thruster suspension, etc. using those frameworks. There exist some (mostly crude) models but there is a huge void concerning experimentally reliable data of typical and non-typical sputter phenomena, which are subject to multiple uncertainties ${ }^{6}$.

With this work, we want to emphasize the need of data in the context of ion sputtering for space applications. In the paper, we show an experimental approach to obtain this data using fairly simple experiments and non-exclusive off-the-shelf equipment. We hope that we can reach out to many more groups and sensitize the EP community to rid the void. Our ultimate goal is to build up a database available to the public with exactly the experimental data needed for (analytical, empirical and heuristic) model validation.

To exemplarily show our proposed strategy, we chose molybdenum and silver as sputtering targets, as they are found in thruster and satellite fabrication respectively and hence, are prone to ion impingement on spacecraft equipped with $\mathrm{EP}^{7}$. We first measured the spatial ion beam distribution of a typical RF ion thruster (RIT-10) and modeled them using an analytical approach. Finally, spatially resolved deposition rates of the chosen sputtering materials with respect to angle of incidence and (primary) energy of the ion beam are obtained using a quartz crystal microbalance (QCM). Another common 
method for the direct analysis of sputter yield data is a weight loss approach ${ }^{8}$. It was not chosen for this study as it does not give information about the ejection angle of sputtered particles, which in turn is crucial regarding the redeposition of material on a spacecraft. In the post process, we validated the results with the established sputtering software framework SDTrimSP, compare Sec. IV B.

\section{THEORETICAL BACKGROUND}

An ion impinging on a surface, subsequently called projectile, potentially transfers its energy and momentum in such a way that an atom is ejected from the target. This process is called sputtering, in contrast to pure ion implantation or backscattering. A projectile undergoes statistical collisions and experiences an energy loss per travelled unit distance. This loss is called stopping power and originates from nuclear (elastic) or electronic (elastic or inelastic) collisions. For low ion energies, nuclear stopping dominates and can be determined with an elastic binary collision approximation (BCA). Particles are hereby treated as hard spheres with Bohr radii, undergoing different binary collisions depending on the mass, energy and collision cross-section. ${ }^{9}$ There are various integration methods based on classical scattering theory to evaluate the scattering angle of atoms after a collision, specified in section IV. Experiments have so far validated computational first order approximations treating polycrystalline materials as amorphous ${ }^{10}$. The energy of sputtered atoms depends on the surface binding energy (SBE) which in turn influences the ejection angle 9 . Considering the total sputtering yield, maxima are generally to be expected for angles of projectile incidence between 50 and 80 degrees ${ }^{10}$.

A QCM makes use of a frequency shift that an oscillator undergoes when a mass is deposited onto its surface. The quartz crystal disc is therefore excited into thickness shear mode vibrations by an external oscillator at a frequency of several MHz. A deposition of sputtered material onto the crystal surface increases the mass of the oscillating system and hence lowers its frequency. Knowing a material's density $\rho\left(\mathrm{g} / \mathrm{cm}^{3}\right)$ and acoustic impedance $z\left(10^{5} \mathrm{~g} / \mathrm{cm}^{2} / \mathrm{s}\right)$ allows the deduction of a deposition rate over time ${ }^{11,12}$. Eq. 1 after Williams et al. ${ }^{13}$ allows the conversion of the measured deposition rate $R(\alpha, \phi)(\mathrm{g} / \mathrm{s})$ to a differential sputtering yield $\gamma(\alpha, \phi)$ (atoms/ion/steradian), making use of the QCM distance to the target (radius $r_{\mathrm{QCM}}$ ) and its position on the hemisphere (polar angle $\alpha$, azimuthal angle $\phi$ ).

$$
\gamma(\alpha, \phi)=\frac{R(\alpha, \phi) N_{\mathrm{A}} q r^{2}}{M J A_{\mathrm{S}}}
$$

$N_{\mathrm{A}}$ is Avogadro's constant, $q$ the elementary charge, $M$ the molar mass of the sputtered atoms, $J$ (A) the ion beam current and $A_{\mathrm{s}}$ the sensor area of the QCM. Eq. 1 is applicable for certain geometrical setups only, which are not present in this study, but it will later be used to deduce a theoretical deposition rate from numerical differential sputtering yield values.

\section{EXPERIMENTAL DESIGN AND METHODOLOGY}

\section{A. Thruster Setup / Beam Characterization}

The electric RF ion thruster (RIT-10, 37 extraction apertures), developed and built at Giessen University, Germany, was set up and operated following conventions and empirical data gained by the respective work group ${ }^{2,14}$. The radio-frequency generator (RFG) as well as the negative and positive high voltages (NHV and $\mathrm{PHV}$ ) were operated as stated in Tab. I. The performance mapping delivered the expected $1 / x$ curve characteristic of an ion thruster when adjusting the RFG power over the mass flow. Grid losses increased noticeably above mass flow rates larger than $1 \mathrm{sccm}$. Furthermore, the findings support the usage of a mass flow above $0.6 \mathrm{sccm}$ to minimize the input power, which led to choosing the operational point of $0.8 \mathrm{sccm}$. Being equipped with a rotary vane pump, a roots pump and a turbo pump, the vacuum chamber is evacuated to $2 \times 10^{-6}$ mbar. The pressure rises to about $6-7 \times 10^{-5}$ mbar in case of a sustained plasma and effective ion extraction (compare Tab. I). This corresponds to a pumping speed of around 6001/s.

Grids of the RIT-10 used in this study are made of titanium (Ti), while alternative materials are molybdenum (Mo) or carbon composites. Grid erosion caused by charge exchange (CEX) ions has to be taken into account, as soon as it contributes a significant amount of atoms to the extracted beam, as this potentially alters the behavior of the beam. For the present thruster no critical fraction of charge-exchange ions has been observed so far when simulating comparable operational points. ${ }^{15}$ Small currents, a low mass flow and a reasonable background pressure in the $10^{-5}$ mbar range support this behavior.

In order to determine the ion beam current during experiments, extracted currents and losses were measured directly on the thruster grids. The evaluation of the grid currents was done with Octave by interpolating the data over time and subtracting the current on the accelerator and the ground grid from the one given by the screen grid. The resulting beam current was averaged, excluding major beam events (peaks in the diagram) by masking the data (compare Fig. 1). Grid losses lie around $7 \%$ but thruster performance was stable and the standard deviation less than $0.1 \mathrm{~mA}$ for total beam currents between $7-9 \mathrm{~mA}$.

A beam analysis with a Faraday cup (Model FC-71A, Kimball Physics Inc.) was carried out to verify the horizontal beam profile. A rotational stage with extended mount (lower swivel arm of QCM setup, Fig. 3) was used, resulting in an absolute distance of $56.6 \mathrm{~cm}$ of the Faraday cup to the thruster grid in perpendicular position. According to this setup, horizontal $180^{\circ}$-scans were conducted along the corresponding radius of $26 \mathrm{~cm}$. Test conditions were varied to gain knowledge about the influence of an active neutralizer (NTR) in contrast to a pure ion beam. 
TABLE I: RIT-10/37 operating conditions during QCM scans for sputtering experiments with silver and molybdenum.

\begin{tabular}{lcc}
\hline \hline Parameter & $1.5 \mathrm{keV}$ Operational Point & $1.8 \mathrm{keV}$ Operational Point \\
\hline RFG Voltage / V & 17.0 & 17.5 \\
\hline NHV / V & -150 & -300 \\
\hline PHV / V & 1500 & 1800 \\
\hline RFG Power / W & 32 & 34 \\
\hline Xe Mass Flow / sccm & 0.8 & 0.8 \\
\hline Beam Current / mA & $7.5 \pm 0.1(\mathrm{Ag})$ & $8.4 \pm 0.1(\mathrm{Ag}) / 8.6 \pm 0.1(\mathrm{Mo})$ \\
\hline Background Pressure / mbar & $\approx 6.1 \times 10^{-5}(\mathrm{Ag})$ & $\approx 6.2 \times 10^{-5}(\mathrm{Ag}) / \approx 6.7 \times 10^{-5}(\mathrm{Mo})$ \\
\hline \hline
\end{tabular}

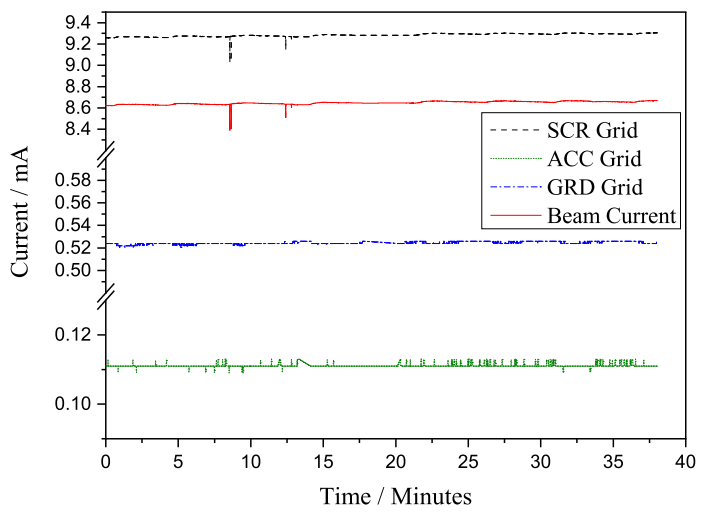

FIG. 1: Exemplary RIT-10/37 grid currents and interpolated resulting beam current (red line, second from the top) during the $1.8 \mathrm{keV}$ sputtering measurement of molybdenum.

Faraday cup scans of the ion beam with and without an active NTR showed a Gaussian current distribution over the radial distance from the beam center line. The maximum of the distribution is slightly eccentrical for both cases by less than two degrees (compare Fig. 2). The Gauss fit of the distribution without the NTR in use gives a standard deviation $(\sigma)$ of $3.75^{\circ}(1.5 \mathrm{keV})$ and $3.42^{\circ}(1.8 \mathrm{keV})$ which result in a divergence angle of $9.18^{\circ}$ and $8.37^{\circ}$ respectively for a $95 \%$ $(2.4477 \sigma)$ interval. The NTR (imitated by two conventional incandescent lamps centered below the extraction grid) influenced the beam current inconsistently and exhibited a slightly less uniform beam profile which is why its use was dismissed during sputtering experiments. Furthermore, a dip in the beam current measurement at the thruster grids was observed parallel to the movement of the Faraday cup through the beam profile.

Lining of the stainless steel chamber walls was realized with graphite plates (Sigraflex expanded graphite) using adhesive Kapton tape (polyimide tape with silicone adhesive). Both materials promise to decrease background contamination during firing of the ion thruster considering their overall lower sputtering yield compared to stainless steel and aluminum.

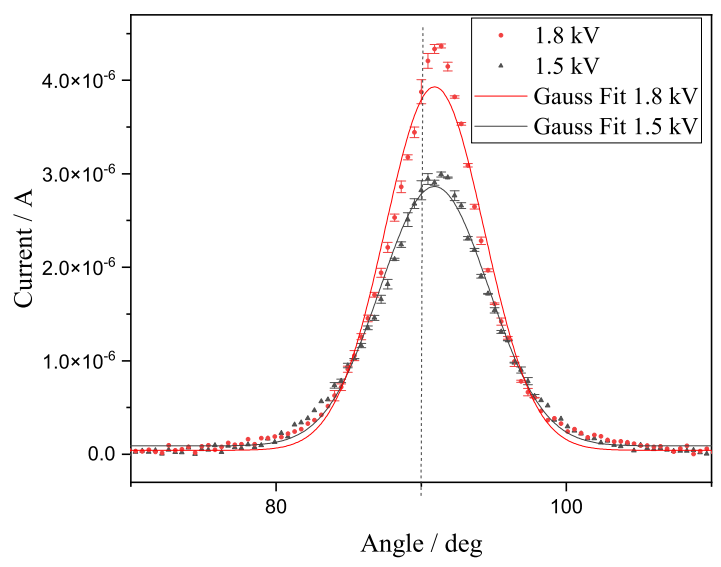

FIG. 2: Beam profile plot from beam current measurements with a Faraday cup for the two chosen thruster operational points of $1.5 \mathrm{keV}$ (lower curve) and $1.8 \mathrm{keV}$ (upper curve).

\section{B. Sputtering Experiments}

Sputtered particles were detected with a QCM sensor head (Intellemetrics Global Ltd.) scanning across a hemisphere over a target's surface. This was realized with a combination of two pivoting arms made of aluminum, each centered on a rotational stage (2-phase stepper motor PRS-110 by PI miCos at base position and URS50-BPPV6 by Newport at upper position), both controlled by one motion controller (ESP301 by Newport). The bi-directional repeatability of incremental motion of the base stage (azimuthal angle, QCM towards and away from the thruster) was given with $0.01^{\circ}$ and observed accordingly. The top stage which addresses the polar angles (QCM up and down) did not exceed an accuracy of $\pm 1^{\circ}$ for its movement but was reset prior to each measurement series. The QCM setup was covered with Kapton tape where feasible in order to decrease background sputtering. A platinum resistor (Pt-1000) was used for temperature observation. It was glued to the QCM mounting bracket, which is in direct contact with the crystal plate, with a two component epoxy thermal conducting glue.

QCM measurements were conducted with sheets of molybdenum (Mo, $99.95 \%$ purity, thickness $0.5 \mathrm{~mm}$ ) and silver (Ag, $99.99 \%$ purity, $1 \mathrm{~mm}$ ), each the size of 10 by $10 \mathrm{~cm}$. 


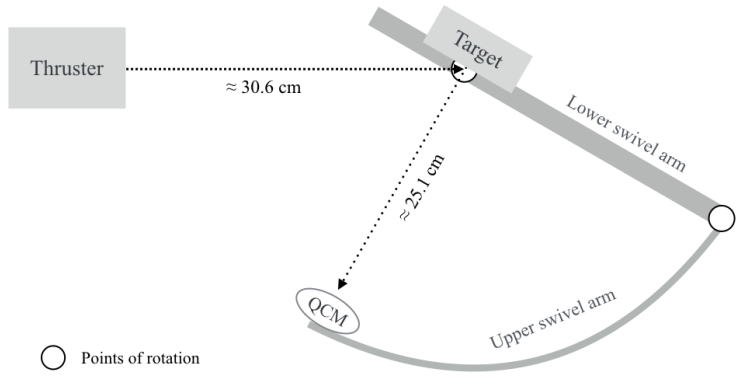

FIG. 3: Schematic drawing of the sputtering setup, top view. QCM exemplarily located at perpendicular position relative to the target. Two points of rotation indicate the possible swivel movement of the arm installed on two rotational stages.

This covers approximately $95 \%$ of the beam profile (compare Sec. V B 1) in a perpendicular target position. However, target materials were positioned under an angle of ion incidence of $60^{\circ} \pm 2^{\circ}$ (defined as off-normal), as this setting promises elevated sputtering yields 7,10 and reduced risk of thruster backsputtering contamination. Due to the Gaussian shape of the beam profile the reduced area of the tilted target $(2.5 \mathrm{~cm}$ along the radial profile of the beam) did not significantly decrease the percentage of incident ions from the initial beam (less than 10\%). The target was located in front of the thruster (see Fig. 4), centered at a distance of $30.6 \mathrm{~cm}$ right above the rotational center of the QCM setup. The QCM sensor was situated at a radial distance $r_{\mathrm{QCM}}$ of $25.1 \mathrm{~cm}$ from the target center. A parking position was arranged to shield the sensor from the direct ion beam and target sputter material when necessary (Fig. 4). Both targets were measured under the $1.8 \mathrm{keV}$ ion energy operational point, while silver was additionally scanned at $1.5 \mathrm{keV}$ (Tab. I). Operational points were named after the estimated peak ion energy, which corresponds to the applied $\mathrm{PHV}^{16}$.

The QCM input parameters of the deposited material's density and acoustic impedance for $\mathrm{Mo}$ and $\mathrm{Ag}$ were $10.2 \mathrm{~g} / \mathrm{cm}^{3}$ and $10.5 \mathrm{~g} / \mathrm{cm}^{3}$ along with $34.3 \times 10^{5} \mathrm{~cm}^{2} / \mathrm{g} / \mathrm{s}$ and $16.7 \times 10^{5} \mathrm{~cm}^{2} / \mathrm{g} / \mathrm{s}$ respectively (according to the manual of the Intellemetrics QCM monitor IL150). The QCM monitor makes use of these values to internally convert the detected frequency shift of the quartz crystal to an accumulated thickness of material per second. The crystal was a plano-convex quartz disc, $14 \mathrm{~mm}$ in diameter and $3 \mathrm{~mm}$ thick which is excited at $6 \mathrm{MHz}$.

Angles of particle ejection (azimuthal and polar angles relative to the target normal) were adapted to the convention used in the SDTrimSP computations (Sec. IV B). The forward $\left(40^{\circ}\right)$, perpendicular $\left(90^{\circ}\right)$ and backward $\left(150^{\circ}\right)$ azimuthal

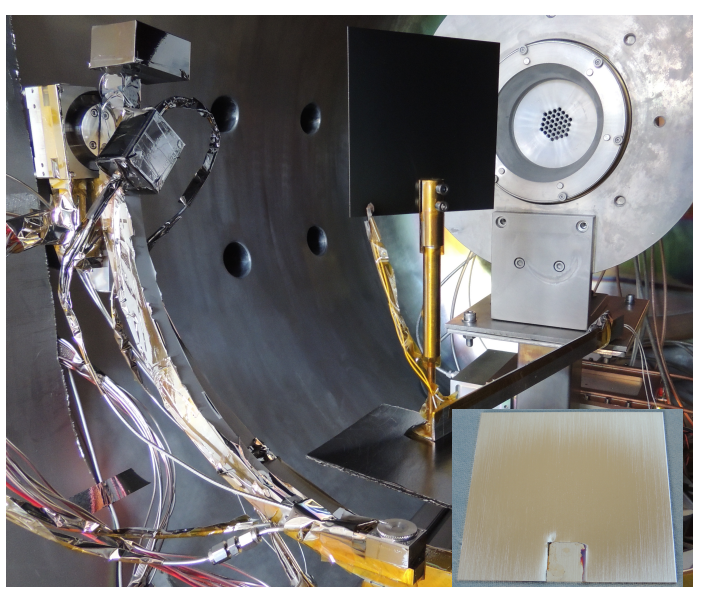

FIG. 4: Mo target setup under $60^{\circ}$ off-normal to the incident ion beam with QCM in parking position. Inset: Irradiation profile on $\mathrm{Ag}$ target after sputtering. The edge regions still exhibit the original brilliance.

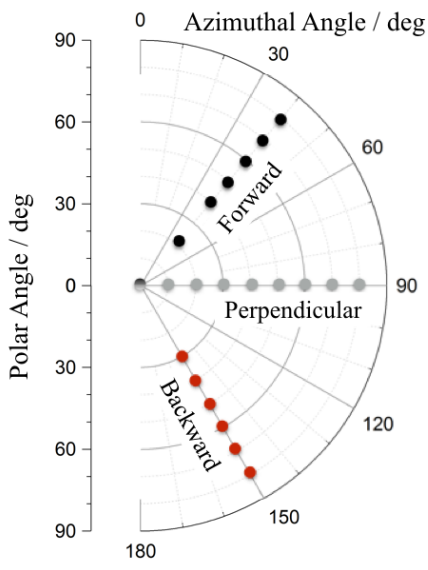

FIG. 5: Sampling points of QCM scans in three different azimuthal sputtering regions (coordinate system adapted to simulations).

sputtering regions for a range of respective polar angles under $10^{\circ}$ steps were chosen to be examined (compare Fig. 5). Polar angles were sufficiently determined ranging from zero (perpendicular ejection) up to just below $90^{\circ}$ (shallow ejection), as the ion beam was approximated as symmetrically identical on the upper and lower half of the target and so was the created sputtering profile. Due to excessive heating of the sensor, the above mentioned points were measured for periods of several seconds only until a sufficient accumulated mass was observed. The corresponding movements were conducted manually via the GUI of the QCM monitor. Subsequently, the increasing absolute thickness signal was 
plotted over time and a deposition rate was derived from its slope.

Additionally, silicon wafer substrates $(5 \times 5 \mathrm{~mm})$ for later element analysis of deposited sputter material by Rutherford Backscattering Spectrometry (RBS) were glued to the inside of the upper arm of the QCM setup with double sided aluminum adhesive tape. The first series of five substrates was installed during pre-testing and was exposed to the ion beam and potentially sputtered thruster and chamber material under various positions for approximately 40 hours. Main waiting times elapsed with the QCM setup in parking position (compare Fig. 4). The second series, installed with the Mo target, was sampled after six hours and 20 minutes and the third series along with $\mathrm{Ag}$ after two hours and 50 minutes of thruster activity, after performed QCM scan routines in each case.

\section{NUMERICAL APPROACH}

\section{A. Beam Current}

To estimate the radial current density $\left(J\right.$ in A $\left./ \mathrm{m}^{2}\right)$ for a $2 \mathrm{D}$ Gaussian beam distribution over radius $r$ at a given distance $z$ from the thruster exit plane, the following expression approximated for an ion beam expanding from a point source was used $^{14}$ :

$$
J=\frac{I_{\mathrm{b}}}{2 \pi \sigma(z)^{2}} \exp \left(\frac{-r^{2}}{2 \sigma(z)^{2}}\right)
$$

with

$$
\sigma=\frac{z}{2.4477} \tan (\beta)
$$

Here, $I_{\mathrm{b}}$ represents the beam current extracted from the thruster, while $\sigma$ gives the according standard deviation. The divergence angle in degrees is noted as $\beta$. The current density calculation is based on Eq. 2. The standard deviation value (2.4477) is chosen according to $95 \%$ of a 2D Gaussian profile projected on a plane perpendicular to the direction of propagation. A cylindrical integration gives the total current $J$ in A over radius $r$ (Eq. 4). The current up to the boundaries implied by the $95 \%$ interval is obtained by setting $R=r-2.4477 \sigma$. The calculation of the current entering the Faraday cup with an orifice of diameter $d$ requires an integration limit of $R=r-d / 2$.

$$
I=2 \pi \int_{0}^{R} r J(r) d r
$$

The generation of CEX ions, along with inelastic collisions of beam ions with neutrals from the background gas present in a vacuum chamber, differs considerably compared to space conditions but is, however, not considered here for the sake of simplicity. For calculations, $I_{\mathrm{b}}$ and $\beta$ were approximated by empirical values of $8.5 \times 10^{-3} \mathrm{~A}$ and $9^{\circ}$ respectively
(Sec.III A). The distance between the thruster and the target as well as the Faraday cup allow a far-field approximation ${ }^{17}$, as they are ten times the grid diameter $d(d \approx 3.1 \mathrm{~cm}$ for the RIT-10/37) or more apart.

\section{B. SDTrimSP}

The simulation program SDTrimSP $(\mathrm{SD}=$ static-dynamic, $\mathrm{SP}=$ sequential and parallel processing) was initialized to run sputtering simulations. The program was published by A. Mutzke et al. ${ }^{18}$ from the Max-Planck-Institute of Plasma Physics, Germany and is based on the Trim code ${ }^{19}$. Theoretical foundations have been well elaborated in W. Eckstein's adaptations of analytical sputtering yields for computer simulations ${ }^{9}$. Models and approximations were chosen based on the findings of $\mathrm{H}$. Hofsäss et al. ${ }^{20}$ and consistently applied for all the SDTrimSP simulations:

- Fully dynamic (Monte Carlo) simulations

- Ion built-up considered

- $\mathrm{KrC}$ interaction potential

- Gauss-Legendre interaction method

- Surface binding model: isbv $=1$

- Bulk binding energy: 0

The beam current density $\left(3.45 \mathrm{~A} / \mathrm{m}^{2}\right)$ and the resulting predicted incident ion fluence onto a target area $\left(0.22 \times 10^{16}\right.$ atoms $\left./ \mathrm{cm}^{2}\right)$ were chosen according to the values estimated at the center of the target with the beam current model in Octave. Simulations were run for molybdenum (thickness $150 \AA$ ) and silver $(170 \AA)$, with xenon ion energies of $1.5 \mathrm{keV}$ and $1.8 \mathrm{keV}$, as well as angles of incidence of $0^{\circ}$ and $60^{\circ}$ for each material. It should be noted that the ion incident angle in SDTrimSP is defined as off normal and is not equal to the azimuthal angles assigned to sputtered particles. The target thickness values were set slightly larger than implantation depths estimated with the program SRIM (The Stopping and Range of Ions in Matter by J.F. Ziegler and J.P. Biersack), in order to avoid transmitted projectiles, as realistic thickness values significantly exceed the simulated thickness for the considered applications.

\section{EXPERIMENTAL RESULTS AND VALIDATION}

\section{A. Experimental Sputtering Results}

During test runs without a sputtering target, the QCM temperature was stable around $19^{\circ} \mathrm{C}$ over a long period including sensor positions away from or facing towards the thruster for different ion energies. Test scans with target material exhibited excessive heating of the water-cooled QCM sensor which is why holding times were kept to a minimum. This is especially true for the forward region, 


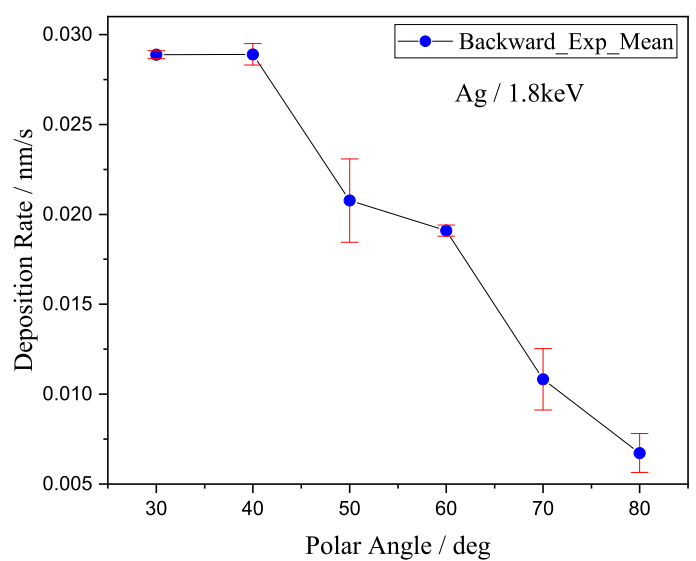

FIG. 6: Good repeatability was achieved when sampling the $\mathrm{Ag}$ deposition rate twice in the backward sputtering region at an ion energy of $1.8 \mathrm{keV}$, angle of ion incidence $60^{\circ}$.

where the QCM temperature eventually exceeded the limit of the Pt-element $\left(105^{\circ} \mathrm{C}\right)$. Due to limitations of the temperature sensor, the target could only be observed for some minutes after thruster ignition, as it heated up from $30^{\circ} \mathrm{C}$ to $105^{\circ} \mathrm{C}$ in less than four minutes. This observation is not crucial, though, as polycrystalline and amorphous materials show mostly no dependence of their sputtering yield on the bulk temperature ${ }^{10}$.

The deposition rates (Fig. 7) generally decrease when moving from perpendicular, i.e. small polar angle, to shallow angles of ejection. Few outliers in the perpendicular azimuthal direction possibly indicate uncertainties of the measurements which were conducted only once due to time restrictions. In the case of silver, the perpendicular azimuthal region exhibits clearly higher deposition rates than the backward and forward regions, while exceeding computational results by up to a factor of four. For molybdenum the effect is less pronounced, as backward and forward sputter rates exhibit roughly the same trend. As the QCM temperature did not rise as quickly in the back sputtering region, two measurements of $\mathrm{Ag}$ under the same conditions were feasible and showed a reasonable repeatability (Fig. 6).

The RBS analysis of coated wafer substrates revealed the composition of the deposited films, but as the setup with the wafer substrates was moved during deposition, no dependence on the spatial position could be deduced from these findings. The film thickness $t_{\mathrm{RBS}}$ in $10^{15} \mathrm{at} / \mathrm{cm}^{2}$ is derived from the fits applied in the RBS analysis software SIMNRA ${ }^{21}$ and calculated to an actual thickness $t_{\text {calc }}$ in $\mathrm{nm}$ from the atomic density in at $/ \mathrm{cm}^{3}$. Mo and $\mathrm{Ag}$ deposits exhibited a noticeable thickness close to $10^{18}$ atoms $/ \mathrm{cm}^{2}$ which corresponds to a few $100 \mathrm{~nm}$. Table II lists the range of film thickness with an exemplarily assumption of deposited, oxidized Mo and $\mathrm{Ag}\left(\mathrm{MoO}_{2}\right.$ and $\mathrm{Ag}_{2} \mathrm{O}$ respectively). The oxidized films were exemplary fitted in two layers with varying oxygen content.
TABLE II: Film thicknesses $t$ and element ratios from RBS evaluation of sputter deposit on silicon wafers. Some samples were analyzed in two layers regarding the oxygen content. Xe and Ti fractions originate from beam ions and sputtered thruster grid material respectively.

\begin{tabular}{lccccc}
\hline \hline Deposit & $t_{\mathrm{RBS}} / 10^{15} \mathrm{at} / \mathrm{cm}^{2}$ & $\mathrm{Mo}_{a t . \%}$ & $\mathrm{Ag}_{a t . \%}$ & $\mathrm{O}_{a t . \%}$ & $t_{\mathrm{nm}}$ \\
\hline $\mathrm{Mo}$ & $304-1449$ & $47-67$ & - & $33-53$ & $150-226$ \\
\hline $\mathrm{MoO}_{2}$ & - & 33.33 & - & 66.66 & $320-483$ \\
\hline $\mathrm{Ag}$ & $492-815$ & - & $58-80$ & $20-42$ & $85-141$ \\
\hline $\mathrm{Ag}_{2} \mathrm{O}$ & - & - & 66.66 & 33.33 & $273-453$ \\
\hline $\mathrm{Xe}$ & $1.2-2$ & - & - & - & $0.9-1.5$ \\
\hline $\mathrm{Ti}$ & $1.6-6$ & - & - & - & $0.4-1.3$ \\
\hline \hline
\end{tabular}

Overall, four samples were measured in each case.

A wafer subjected only to chamber background sputtering and xenon ion beam irradiation during pre-testing revealed equally small amounts of xenon and titanium, smaller than $1 \%$ of the atoms deposited during sputtering. An exemplary conversion of the $1.3 \mathrm{~nm}$ titanium deposit with an atomic radius of $140 \mathrm{pm}$ gives around ten atomic layers.

\section{B. Numerical Validation}

\section{Beam Properties}

The beam current model (Eq. 2) gives a linearly decreasing beam current density with a value of $3.45 \mathrm{~A} / \mathrm{m}^{2}$ at the center line and $z=30.6 \mathrm{~cm}$, representing the position of the target center. The beam distribution in this plane has a $\sigma$ of $1.98 \mathrm{~cm}$, resulting in an area of $94.09 \mathrm{~cm}^{2}$ impinged by $95 \%$ of the beam current.

A comparison of the current calculated at $z=56.6 \mathrm{~cm}$ (position and orifice size of the Faraday cup, initial ion beam current $11.7 \times 10^{-3} \mathrm{~A}, 1.5 \mathrm{keV}$ thruster setting) to the current measured with the Faraday cup under said conditions, allows an estimation of the acceptance of its orifice. The computational beam current entering the sensor is $27.2 \times 10^{-6} \mathrm{~A}$, while the experimental value measured in the center line of the beam gives $2.99 \times 10^{-6} \mathrm{~A}$, resulting in a low transmission of approximately $11 \%$.

\section{SDTrimSP}

The angular distribution of ejected target atoms was divided into counts per solid angle with a two-dimensional binning function across the surface of a quarter-sphere (radius $r_{\mathrm{QCM}}=25.1 \mathrm{~cm}$, symmetrically identical to second half of hemisphere). The corresponding polar contour plots are available as supplementary material (Fig. S1). All case studies tend towards very low sputtering along the contour of the polar plot, which equals an ejection under large and hence shallow polar angles. The distribution is shifted to the forward sputtering region for an angle of incidence of $60^{\circ}$ in each case. 
In order to compare simulation results to experimental data, the binning process described above was further used to calculate a differential sputtering yield per steradian from the computational data. Therefore, the counts within bins which correspond to the experimentally measured points on the hemisphere (compare Fig. 5, QCM sensor head equals $4.44 \times 10^{-4}$ str) were used. The differential yield in turn was used to deduce the deposition rate (comparable to $R$ in Eq. 1) according to the experimental setup, using the impinging ion current at the center of the target $(3.05 \mathrm{~mA}$ at $1.5 \mathrm{keV}$ and $3.45 \mathrm{~mA}$ at $1.8 \mathrm{keV})$. The resulting angular dependences of the deposition rates are plotted in Fig. 7 in comparison to experimental data. As the backsputtering region could not be measured up to a perpendicular polar angle $\left(0^{\circ}\right)$ in experiment, the computational data is left out accordingly.

\section{DISCUSSION}

Two stable operational points of the RIT-10/37 were iteratively determined and sputtering measurements successfully conducted with a silver and a molybdenum target. Experimental and computational results lie in the same order of magnitude, following similar trends. However, the RBS analysis of sputter deposit revealed the formation of oxides and excessive heating of the QCM sensor head was observed.

Experimental data follow the trend of computational SDTrimSP results well, with rates decreasing towards large polar angles. Overall higher experimental values have been observed for perpendicular azimuthal regions. For non-normal ion incidence $\left(60^{\circ}\right.$ in this study), an over-cosine distribution tilted in the forward direction is expected, with sputter rates increasing from backward, to perpendicular, to forward ejection $^{22}$. Simulations also give an estimate of a sputtering plume shifted towards forward sputtering in comparison to perpendicular ion incidence (see Supplementary Material, S1). Williams et al. ${ }^{13}$, however, found that for Mo this behavior reverts to a nearly symmetric distribution around the target normal.

Contradictions of differing values from literature, simulation and experiment demand for a rigorous evaluation of contributing effects. Resputtering due to the direct ion beam, which is favored in the forward sputtering region, is one possible reason that may have artificially lowered the measured deposition rates in the forward region. Additionally, a decrease in the effective sticking coefficient of sputtered metallic particles can be caused by thermal desorption, which is facilitated if the substrate (here: QCM crystal) is heated due to incoming energy flux ${ }^{23}$. This demands for a more sophisticated cooling system. Moreover, the effective sticking coefficient is lowered when resputtering or reflection occur, which in turn are favored for high-energy particles.

The energy of sputtered atoms is based on various factors, but mainly on their angle of ejection for incoming ion energies above $1 \mathrm{keV}$. In general, off-normal ejection results in higher mean energy, while peak ejection energies rise only slightly compared to perpendicular or shallow ejection ${ }^{9}$. Furthermore,

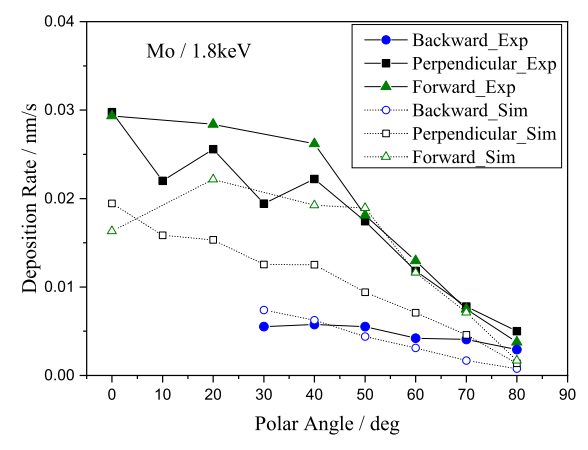

(a) Mo at an ion energy of $1.8 \mathrm{keV}$.

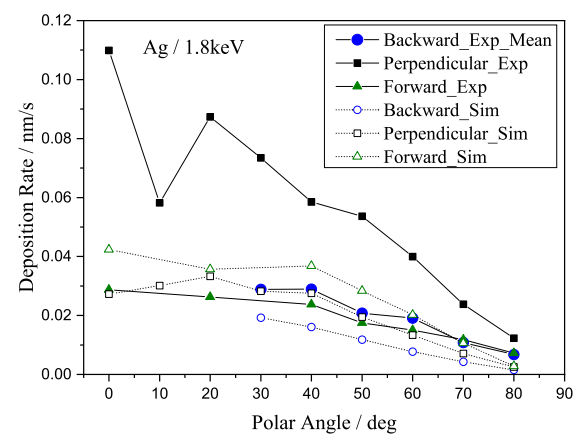

(b) Ag at an ion energy of $1.8 \mathrm{keV}$.

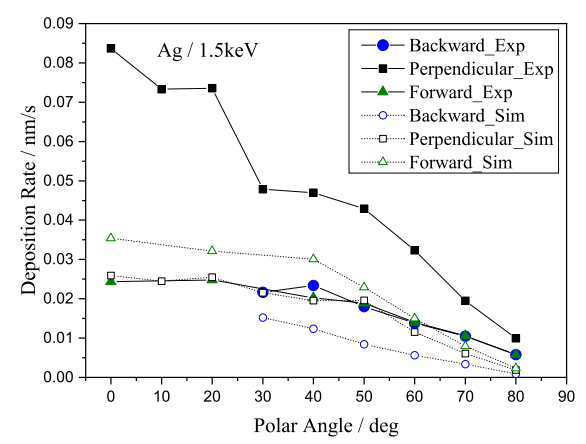

(c) $\mathrm{Ag}$ at an ion energy of $1.5 \mathrm{keV}$.

FIG. 7: Computational (SDTrimSP, dotted lines) and experimental (QCM, solid lines) deposition rates for different azimuthal regions over the polar angle (compare Fig. 5). Comparison of Mo and Ag sputtered at an ion energy of $1.8 \mathrm{keV}(\mathrm{a}, \mathrm{b})$, as well as $\mathrm{Ag}$ at $1.5 \mathrm{keV}$ (c), all sputtered under an angle of ion incidence of $60^{\circ}$. Good repeatability was achieved when testing $\mathrm{Ag}$ in the backward region (b), see

Fig. 6 for details.

the heavy mass of Xe ions in general favors the generation of secondary knock-on atoms, while the shallow ion incidence used in this study $\left(60^{\circ}\right)$ in turn promotes high energy primary knock-on atoms 9 . Hence, the effective sticking coefficient is subject to change depending on the region of detection as well as to the experimental setup in general. 
In the context of SDTrimSP, care should be taken regarding the assumption of the surface binding energy (SBE), as the program does not take into account an initial surface roughness or a change of SBE during the sputtering process. In terms of experimental investigations, an analysis of the target surface before and after experiments could reveal the formation of patterns, which can in turn favor preferred sputtering orientations ${ }^{24}$. In order to achieve a good comparison of experiment and simulations, a thorough knowledge of the ion beam impacting the target surface is crucial. Furthermore, the geometrical setup could be improved in the test chamber to create conditions that allow a conversion of measured deposition rates to a sputtering yield per solid angle ${ }^{13,25}$.

RBS analysis was intended for qualitative analysis of sputter deposit to adapt the settings of the QCM monitor, which relies on an accurate preset film density and acoustic impedance. The findings of deposited oxides instead of pure sputtered metals indicate that measured deposition rates are presumably too low regarding an oxide film thickness. The formation of oxides revealed by RBS analysis was to be expected, as atomic oxygen formed by the RF-plasma inside the ground-based vacuum chamber favors this process. ${ }^{26}$.

Background chamber pressure in the low $10^{-5}$ mbar range was achieved during thruster activity which is equivalent to comparable experiments found in literature ${ }^{7,13}$. However, the performance of the facility could be further improved by reducing the base pressure in order to reduce the process of oxide formation. Beam events ceased to appear regularly after a longer run time of the thruster and can most probably be explained with discharge due to grid contamination, but also with artifacts of the membrane in the xenon mass flow controller or insufficient grounding of the facility.

The ion beam characterization with a Faraday cup revealed the expected Gaussian beam profile but the measured profile might be artificially narrowed by the acceptance angle of the Faraday cup, so the actual divergence angle could be slightly bigger than determined. Furthermore, a dip in grid currents observed during the Faraday cup scans emphasizes the invasiveness regarding beam properties when positioning setup parts in the beam. A non-invasive beam characterization would be needed in order to conduct simultaneous measurements during sputtering experiments.

\section{CONCLUSION}

This study addressed the need of the EP-community to generate spatially resolved sputtering data, by implementing an experimental setup with readily available tools along with a computational validation using the program SDTrimSP. The targeted QCM-analysis was successfully conducted on molybdenum and silver, sputtered by the xenon ion beam of a RIT-10. Results seem reasonable, as experiments and simulations lie in the same order of magnitude while exhibiting comparable trends over the analyzed spatial angles. Deviations, especially in the perpendicular azimuthal regions, demand for future investigations. Moreover, a temperature calibration and sufficient cooling of the QCM sensor is needed with regard to the significant heating observed. The latter is likely to cause frequency instabilities and influence the sticking coefficient and is most commonly induced by bombarding ions when operating a QCM in a sputtering $\operatorname{setup}^{27}$. Therefore, not only the quantitative but also the energetic distribution of sputtered particles would be of interest here. Both information are available in the program SDTrimSP.

The findings of oxides by RBS analysis demand an initial deposit characterization (composition, density, acoustic impedance) in order to adapt the QCM monitor settings. Furthermore, the sampling procedure must be improved to rule out dependencies on sampling orders. A stable thruster operation was realized, creating a symmetric, roughly centered beam, ruling out dependencies in this matter.

In order to examine realistic scenarios for satellite applications, a more accurate knowledge of the ion beam distribution over a (tilted) target surface is needed, especially if the target itself alters the beam properties by inhibiting its free expansion. Another relevant factor is to understand the dependence of the aforementioned surface roughness on the sputter yield and distribution, particularly if the surface structure changes significantly over longer time scales. Optical coatings as they are used in solar cell technologies often have a special design in terms of surface patterning which should be taken into account when testing or simulating the sputtering behavior of such surfaces.

Overall, the subject of sputtering in space applications is prone to multiple uncertainties ${ }^{6}$, ranging from the approximations made in computational approaches, over experimental data acquisition, to the assessment of actual applications. We hope that this work emphasizes the need and advocates the research of experimental sputtering data for space applications by presenting the corresponding difficulties as well as possibilities in terms of experimental work and computational supplements.

\section{SUPPLEMENTARY MATERIAL}

See "make this a link" for the polar contour plots of the SDTrimSP angular sputtering distribution simulation (Sec. VB 2).

\section{ACKNOWLEDGMENTS}

This work was conducted at DLR (German Aerospace Center), Institute of Aerodynamics and Flow Technology, Spacecraft Department in Göttingen, Germany. The construction of the sensor mount and preliminary positioning unit was developed at DLR as preparatory work by Jonas Schäfer. Advice for electronical matters was gratefully received from C. Geile at DLR. The RBS analysis of the wafer substrates was kindly 
conducted at University of Göttingen, Institute of Nuclear and Particle Physics, supported by H. Hofsäss, who also provided a helpful introduction and kind support for SDTrimSP simulations.

\section{AIP PUBLISHING DATA SHARING POLICY}

The data that support the findings of this study are available from the corresponding author upon reasonable request.

\section{REFERENCES}

${ }^{1}$ S. Mazouffre, "Electric propulsion for satellites and spacecraft: established technologies and novel approaches," Plasma Sources Science and Technology 25, 033002 (2016).

${ }^{2}$ K. Holste, P. Dietz, S. Scharmann, K. Keil, T. Henning, D. Zschätzsch, M. Reitemeyer, B. Nauschütt, F. Kiefer, F. Kunze, J. . Zorn, C. Heiliger, N. Joshi, U. Probst, R. Thüringer, C. Volkmar, D. Packan, S. Peterschmitt, K.-T. Brinkmann, H.-G. Zaunick, M. H. Thoma, M. Kretschmer, J. Leiter, S. Schippers, K. Hannemann, and P. J. Klarl, "Ion thrusters for electric propulsion: Scientific issues developing a niche technology into a game changer," Review of Scientific Instruments 91, 061101 (2020).

${ }^{3}$ D. M. Goebel and I. Katz, Fundamentals of Electric Propulsion: Ion and Hall Thrusters, JPL space science and technology series (Wiley, 2008).

${ }^{4}$ R. S. Robinson and S. Rossnagel, "Sputtering phenomena in ion thrusters," Tech. Rep. (Lewis Research Center, National Aeronautics and Space Administration, 1983).

${ }^{5}$ M. Grabe and C. E. Soares, "Status and future of research on plume induced contamination," in Proceedings of the International Astronautical Congress, IAC (IAF, 2019).

${ }^{6} \mathrm{~J}$. T. Yim, "A survey of xenon ion sputter yield data and fits relevant to electric propulsion spacecraft integration," in 35th International Electric Propulsion Conference (Georgia Institute of Technology, Atlanta, Georgia, 2017).

${ }^{7}$ M. Tartz, T. Heyn, C. Bundesmann, C. Zimmermann, and H. Neumann, "Sputter yields of mo, ti, w, al, ag under xenon ion incidence," The European Physical Journal D 61, 587-592 (2011).

${ }^{8}$ O. Girka, O. Bizyukov, S. Bogatyrenko, and I. Bizyukov, "Aluminium surface morphology behaviour under high-flux helium ion bombardment," Nuclear Instruments and Methods in Physics Research Section B: Beam Interactions with Materials and Atoms 405, 31-35 (2017).

${ }^{9} \mathrm{~W}$. Eckstein, Computer simulation of ion-solid interactions, Springer series in materials science, Vol. 10 (Springer, 1991).

${ }^{10} \mathrm{R}$. Behrisch and W. Eckstein, Sputtering by Particle Bombardment: Experiments and Computer Calculations from Threshold to MeV Energies, Topics in Applied Physics (Springer, 2007).
${ }^{11} \mathrm{C} . \mathrm{Lu}$ and A. Czanderna, Applications of Piezoelectric Quartz Crystal Microbalances, Methods and Phenomena (Elsevier Science, 2012).

${ }^{12}$ J. G. Miller and D. I. Bolef, "Acoustic wave analysis of the operation of quartz-crystal film-thickness monitors," Journal of Applied Physics 39, 5815-5816 (1968).

${ }^{13}$ J. D. Williams, M. M. Gardner, M. L. Johnson, and P. J. Wilbur, "Xenon sputter yield measurements for ion thruster materials," in 28th International Electric Propulsion Conference (2004).

${ }^{14}$ C. Volkmar, C. Geile, A. Neumann, and K. Hannemann, "Direct-current current transformer for the measurement of an electric propulsion ion beam," Review of Scientific Instruments 90, 033303 (2019).

${ }^{15}$ A. Reeh, U. Probst, and P. J. Klar, "Global model of a radio-frequency ion thruster based on a holistic treatment of electron and ion density profiles," The European Physical Journal D 73, 232 (2019).

${ }^{16}$ A. Bulit, J. P. Luna, J. G. Del Ámo, B. Lotz, D. Feili, and H. Leiter, "Experimental investigations on the influence of the facility background pressure on the plume of the rit-4 ion engine," in Proceedings of the 32nd International Electric Propulsion Conference, IEPC-2011-028, Wiesbaden, Germany (2011).

${ }^{17} \mathrm{~T}$. Reynolds, "Mathematical representation of current density profiles from ion thrusters," in AIAA/SAE 7th Propulsion Joint Specialist Conference (American Institute of Aeronautics and Astronautics, 1971).

${ }^{18}$ A. Mutzke, R. Schneider, W. Eckstein, and R. Dohmen, "SDTrimSP Version 5.00," IPP Report (2011).

${ }^{19}$ J. F. Ziegler and J. P. Biersack, "The stopping and range of ions in matter," in Treatise on Heavy-Ion Science: Volume 6: Astrophysics, Chemistry, and Condensed Matter (Springer US, 1985) pp. 93-129.

${ }^{20} \mathrm{H}$. Hofsäss, K. Zhang, and A. Mutzke, "Simulation of ion beam sputtering with sdtrimsp, tridyn and srim," Applied Surface Science 310, 134-141 (2014).

${ }^{21}$ M. Mayer, "Simnra, a simulation program for the analysis of nra, rbs and erda," AIP Conference Proceedings 475, 541-544 (1999).

${ }^{22} \mathrm{~T}$. Lautenschläger and C. Bundesmann, "Reactive ion beam sputtering of ti: Influence of process parameters on angular and energy distribution of sputtered and backscattered particles," Journal of Vacuum Science \& Technology A: Vacuum, Surfaces, and Films 35, 041001 (2017).

${ }^{23}$ S. Mahieu, K. Van Aeken, D. Depla, D. Smeets, and A. Vantomme, "Dependence of the sticking coefficient of sputtered atoms on the targetsubstrate distance," Journal of Physics D: Applied Physics 41, 152005 (2008).

${ }^{24}$ H. Hofsäss, O. Bobes, and K. Zhang, "Is sputtering relevant for ion-induced self-organized pattern formation?" in AIP Conference Proceedings, Vol. 1525 (American Institute of Physics, 2013) pp. 386-391.

${ }^{25}$ A. Yalin, B. Rubin, S. Domingue, Z. Glueckert, and J. Williams, "Differential sputter yields of boron nitride, quartz, and kapton due to low energy xe+ bombardment," in 43rd AIAA/ASME/SAE/ASEE Joint Propulsion Conference \& Exhibit (American Institute of Aeronautics and Astronautics, 2007).

${ }^{26}$ A. De Rooij, “The oxidation of silver by atomic oxygen,” ESA Journal 13, 363-382 (1989).

${ }^{27}$ B. Rubin, J. L. Topper, C. C. Farnell, and A. P. Yalin, "Quartz crystal microbalance-based system for high-sensitivity differential sputter yield measurements," Review of Scientific Instruments 80, 103506 (2009). 